\title{
Growth of $\mathrm{Ag}$ icosahedral nanocrystals on a $\mathrm{SrTiO}_{3}(001)$ support
}

\author{
Fabien Silly and Martin R. Castell ${ }^{\text {a) }}$ \\ Department of Materials, University of Oxford, Parks Road, Oxford OX1 3PH, United Kingdom
}

(Received 1 August 2005; accepted 4 October 2005; published online 15 November 2005)

\begin{abstract}
We have investigated the structure and morphology of self-assembled silver nanocrystals supported on a $\mathrm{SrTiO}_{3}(001)-(2 \times 1)$ substrate using scanning tunneling microscopy. Ag forms nanocrystals with five-fold symmetry which have an icosahedral shape. Nanocrystals with point, edge, and face orientation (five-fold, two-fold, and three-fold symmetry, respectively) have been studied. The images of these nanocrystals allow a crystallographic identification of the supported shape of the icosahedral form. (C) 2005 American Institute of Physics. [DOI: 10.1063/1.2133909]
\end{abstract}

Self-assembled and self-organized silver nanocrystals with controlled shapes and sizes are a focus of research due to their potential technological significance. Silver nanoparticles have a strong surface plasmon resonance in the visible region of the optical spectrum with a large effective scattering cross section. This makes them suitable for applications in nano-optics, ${ }^{1-5}$ or for chemical sensing via surfaceenhanced Raman scattering (SERS).

An exciting possibility offered by processing metals on the nanoscale is the creation of nanocrystals with shapes and crystal structures that are not feasible on macroscopic length scales. One example of this is the formation of multiply twinned crystals with five-fold symmetry. Predicted in $1931,{ }^{8}$ five-fold twinned structures were first observed in $1957 .{ }^{9}$ For these particles, the bulk atoms are not in their lowest-energy configurations, but their surface atoms are in more favorable positions. Therefore, for sufficiently small particles with a large surface area-to-volume ratio, multiply twinned particles may have the atomic configuration with the lowest overall free energy. Calculations on silver nanocrystals have shown that multiply twinned icosahedrons are the lowest energy shape for small volumes, ${ }^{10}$ but kinetic factors can cause icosahedral nanocrystals to retain their form even when they are significantly larger. ${ }^{11}$

Many applications for the use of Ag nanocrystals require that they are attached to a support, but most studies to date have only been concerned with fully formed twinned particles. The important influence of the substrate, and the effect it has on the nanocrystal shape, tend to be ignored. However, for twinned particles nucleated on a support, the interaction between the particle and the substrate is a key factor.

In this letter, we report on a scanning tunneling microscopy (STM) study of Ag nanocrystals grown on singlecrystal $\mathrm{SrTiO}_{3}(001)$. The STM images show that Ag nanocrystals adopt five-fold symmetry when deposited on a substrate heated to $250{ }^{\circ} \mathrm{C}$. These nanocrystals have a supported icosahedral shape, that adopt three distinct orientations, presenting a two-, three-, or five-fold symmetry top surface. A detailed characterization of the nanocrystal morphology allows us to identify the supported equilibrium shape of the icosahedral nanocrystals.

As our support for the growth of Ag nanocrystals, we use a $\mathrm{SrTiO}_{3}(001)$ surface. $\mathrm{SrTiO}_{3}$ crystallizes into the cubic perovskite structure with a $3.905 \AA$ lattice parameter, and its

${ }^{a)}$ Electronic mail: martin.castell@materials.ox.ac.uk
(001) surface presents a multitude of different reconstructions depending on sample preparation. ${ }^{12,13}$ The surface of this oxide was previously successfully used for the growth over macroscopic scales of regular metal nanocrystals of various shapes. $^{14-17}$

In its pure form, $\mathrm{SrTiO}_{3}$ has a $3.2 \mathrm{eV}$ band gap which would make it unsuitable for imaging in the STM. To overcome this problem, we use crystals doped with $0.5 \%$ (weight) $\mathrm{Nb}$. The crystals were epipolished (001) and supplied by PI-KEM, U.K. $\mathrm{SrTiO}_{3}(001)-(2 \times 1)$ reconstructed surfaces were prepared by sputtering the $\mathrm{SrTiO}_{3}$ samples with argon ions and subsequently annealing them in an ultrahigh vacuum (UHV) at $600{ }^{\circ} \mathrm{C}$ for $9 \mathrm{~h}$. We deposited $\mathrm{Ag}$ from an electron-beam evaporator (Oxford Applied Research EGN4) using $99.95 \%$ pure Au rods supplied by Goodfellow, U.K. Our STM is manufactured by JEOL (JSTM 4500s) and operates in UHV $\left(10^{-8} \mathrm{~Pa}\right)$. We used etched $\mathrm{W}$ tips to image the samples at room temperature with a bias voltage applied to the sample.

Figure 1 shows the topography of the $\mathrm{SrTiO}_{3}(001)-(2$

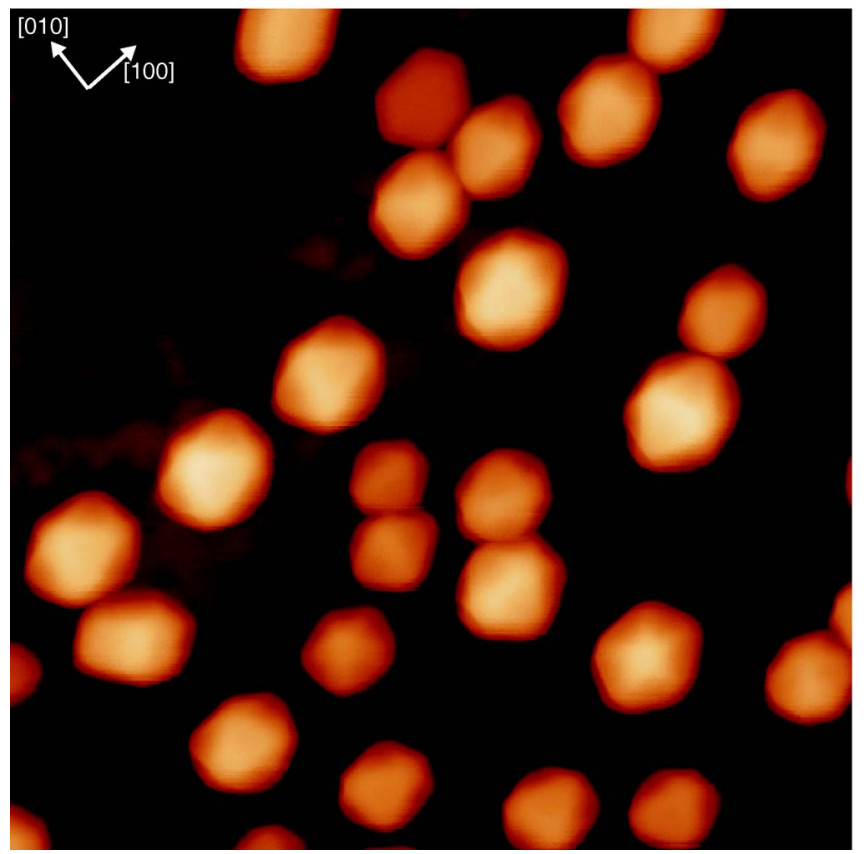

FIG. 1. (Color online) $\mathrm{Ag}$ deposition onto a $250{ }^{\circ} \mathrm{C} \mathrm{SrTiO}_{3}(001)-(2 \times 1)$ substrate gives rise to five-fold symmetry nanocrystals (image size 80 $\left.\times 80 \mathrm{~nm}^{2}\right) ;\left(V_{s}=+4.0 \mathrm{~V}, I_{t}=30 \mathrm{pA}\right)$. 
(a)
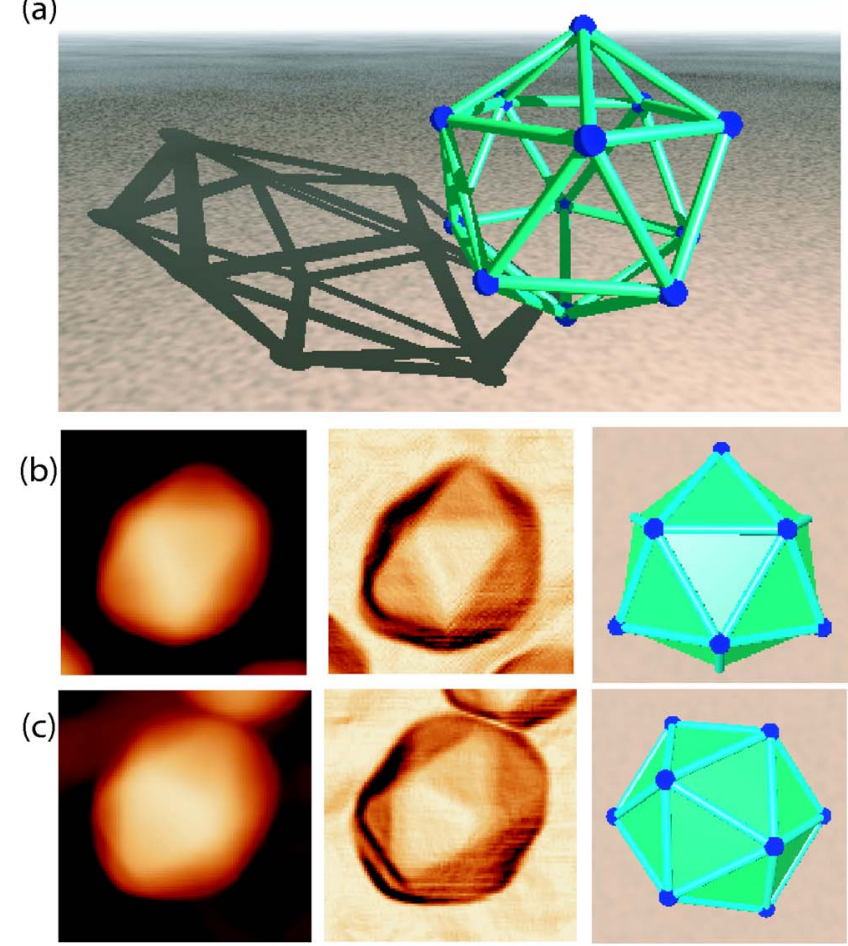

(d)
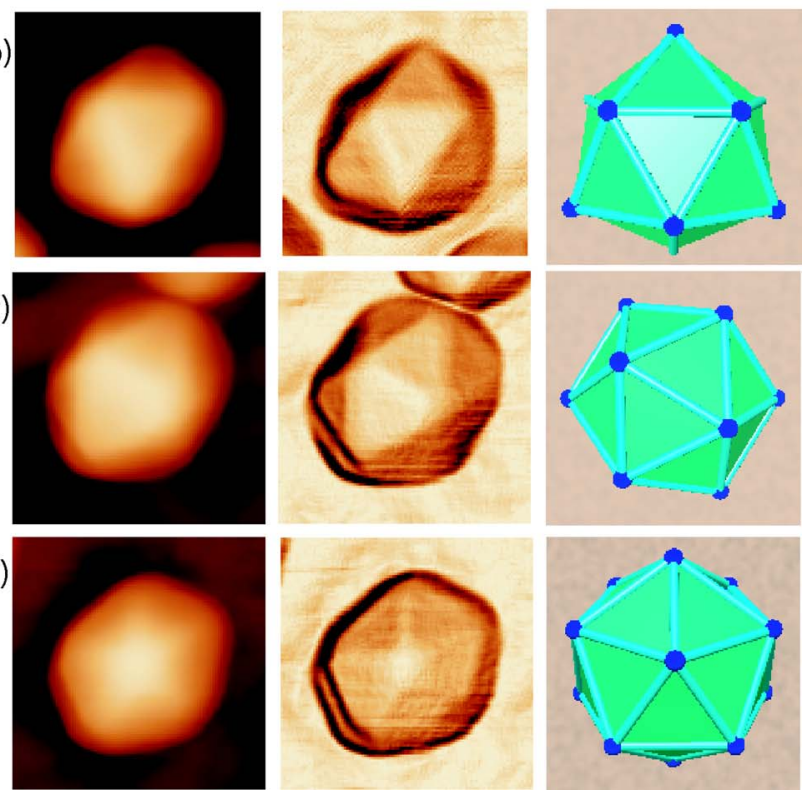

FIG. 2. (Color online) (a) Three-dimensional representation of an icosahedron. Supported icosahedral nanocrystals in the (b) face orientation, (c) edge orientation, and (d) point orientation. The panels on the left show topographic STM images (image sizes $15 \times 15 \mathrm{~nm}^{2} ; V_{s}=+4.0 \mathrm{~V}, I_{t}=30 \mathrm{pA}$ ). The central panels show derivative STM images to better illustrate their shapes. The right-hand panels show models of the various supported icosahedral orientations.

$\times 1)$ surface following Ag deposition on a substrate heated to $250{ }^{\circ} \mathrm{C}$. Ag forms well-defined nanocrystals on the surface. The most commonly observed nanocrystal shape (55\%) has a flat triangular top surface (three-fold symmetry). The second most commonly observed shape $(35 \%)$ has a ridge separating two triangular facets (two-fold symmetry). The third most commonly observed shape (9\%) has a pyramidal form with five side facets (five-fold symmetry). In all of these nanocrystals, each point is formed by the junction of five facets. We should also mention that an additional nanocrystal shape was observed. It has a truncated triangle top surface and is the supported single-crystal face-centered-cubic (fcc) Wulff shape with a (111) interface, but less than $1 \%$ of the nanocrystals have this shape.

The Ag nanocrystals in Fig. 1 can be compared to icosahedral particles. An icosahedron [Fig. 2(a)] has six five-fold axes, and is composed of 20 tetrahedra sharing one common point at the center (point group symmetry $\mathrm{I}_{h}$ ) which is bounded by 20 triangular faces. Three possible high symmetry orientations for supported icosahedral particles are experimentally observed as mentioned previously and shown in Figs. 2(b)-2(d). In Fig. 2(b), the nanocrystal has a face as its top surface which is parallel to the substrate plane, and which we term "face orientation." In Fig. 2(c), the nanocrys-
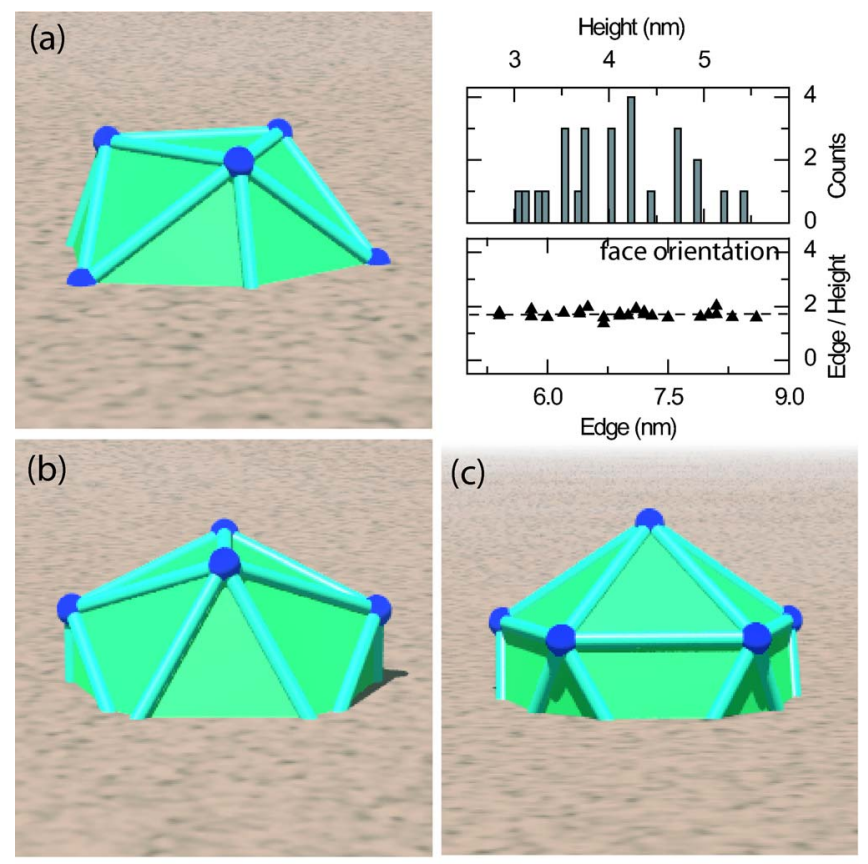

(c)
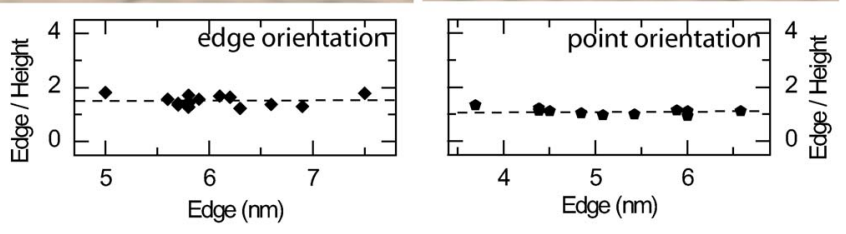

FIG. 3. (Color online) (a) Schematic model of a supported icosahedral nanocrystal with face orientation. The intersection of the icosahedron with the substrate plane corresponds to our experimental measurements. To the right of the model, there is a histogram showing the quantization of the face orientation nanocrystals heights. Below, it is a plot of the edge to height ratio as a function of edge length. (b) Model of a supported icosahedral nanocrystal with edge orientation. The plot below shows the constant edge to height ratio. (c) Model of a supported icosahedral nanocrystal with point orientation, and edge to height ratio plot below.

tal has an edge at the top which is parallel to the substrate plane, and which we term "edge orientation." In Fig. 2(d), the nanocrystal has a point at the top and we term this "point orientation". Detailed measurements of the angle between two triangular facets for the face and edge orientations yields $140 \pm 4^{\circ}$, which is close to the $138.2^{\circ}$ angle of an ideal icosahedron. We should add that the icosahedral nanocrystals also sometimes deviate from their ideal face, edge, and point orientations. ${ }^{18}$ Furthermore, there does not appear to be a single epitaxial relationship between the three supported shapes and the substrate. In many images, we notice that neighboring nanocrystals of the same shape also have the same rotational relationship. However, as we do not know the crystallography of these multiply twinned particles, we cannot determine the interface crystallography.

The face orientation nanocrystal heights are quantized into steps of $2.35 \AA$ [Fig. 3(a)], close to the $2.36 \AA$ (111) interplanar spacing of Ag. The edge length $(\ell)$ to height $(h)$ ratios of the icosahedral nanocrystals as a function of edge length are shown in Fig. 3 for the three orientations. This ratio is observed to be constant for the three orientations, showing that their shape is independent of size. The constant ratio is $\ell / h=1.71 \pm 0.14$ for the face orientation, $\ell / h$ $=1.49 \pm 0.19$ for the edge orientation, and $\ell / h=1.11 \pm 0.09$ for the point orientation. The error in the ratio denotes the

standard deviation of the measurements. 
For the face orientation, the experimental value of $\ell / h$ is 1.71, which corresponds to the shape shown in Fig. 3(a). This shape has four fully apparent facets and six partial facets. For the edge orientation $\ell / h=1.49$. This shape is shown in Fig. 3(b) and has two fully apparent facets and ten partial facets. For the point orientation, the experimental value of $\ell / h$ is 1.11 , which corresponds to the shape shown in Fig. 3(c). This shape has five fully apparent facets and ten partial facets.

Typically, the edge lengths of the icosahedral nanocrystals were between 4 and $9 \mathrm{~nm}$, corresponding to volumes of around $60 \mathrm{~nm}^{3}$ and $700 \mathrm{~nm}^{3}$, respectively. The largest supported icosahedron observed had a $15 \mathrm{~nm}$ edge $\left(3300 \mathrm{~nm}^{3}\right.$ volume).

The formation of five-fold twinned structures has been a matter of some debate. Five-fold twinned structures, in principle, may be found in any crystalline material which allows twinning on alternate coplanar twin planes enclosing an angle of about $2 \pi / 5$. fcc crystals which exhibit a low twin boundary energy can favor growth shapes deviating from the single-crystalline Wulff shape. Experimental evidence of this has been obtained for Ag. ${ }^{19}$ A multiply twinned particle is bounded by triangular (111) faces of tetrahedra only. The icosahedral shape presents only (111) facets in comparison to the fcc truncated octahedron, and may thereby realize a more effective minimization of surface energy. The nanocrystal shape therefore depends on the balance of the twinning energy versus the low index facet surface energies. The formation of icosahedral structures is generally attributed to a nucleation and layer-by-layer growth process. ${ }^{20,21}$

Until now, studies only considered icosahedral particles as free particles consisting of 20 twin-related tetrahedra arranged around a common point. This is valid in the case of unsupported particles, but for supported particles the interface plane with the substrate cuts the icosahedral shape as shown in Fig. 3. We know that the particles sit on the surface and are not partially buried ${ }^{22}$ because the STM tip could be used to dislodge the icosahedral nanocrystals. No remaining pit or other traces were observed that would indicate buried nanocrystals. The interaction with the substrate is therefore critical in determining the shape and orientation of the nanocrystals.

The calculations of Mottet et al. ${ }^{10}$ on free Ag nanocrystals indicate that below volumes of around 200 atoms, the icosahedral form is the most thermodynamically stable. Between 200 and 20000 atoms, the Marks decahedron ${ }^{23}$ has the lowest energy. Above 20000 atoms, the fcc truncated octahedron shape is the most stable. In our studies, we have investigated nanocrystals with volumes up to around 200000 atoms and found that the vast majority have an icosahedral shape. It is therefore reasonable to conclude that our nanocrystals nucleated as supported icosahedrons, and retained this shape even when they grew beyond its thermodynamic stability. Thus, all the icosahedral nanocrystals in our study are growth shapes rather than thermodynamic equilibrium shapes.

In summary, we have investigated Ag nanocrystal growth on a $\mathrm{SrTiO}_{3}(001)-(2 \times 1)$ support. Our results show that $\mathrm{Ag}$ forms five-fold symmetric particles. These particles are supported icosahedral nanocrystals with three preferred orientations. We believe that the nanocrystals are a growth shape that evolved from small icosahedral nuclei. Nanocrystals of this type are expected to have different properties from their single-crystal counterparts and may therefore be of use in novel chemical and optical applications.

The authors would like to thank the Royal Society and DSTL for funding, and Chris Spencer (JEOL U.K.) for valuable technical support. They are also grateful to John A. Venables and L. D. Marks for illuminating discussions.

${ }^{1}$ F. Silly, A. O. Gusev, A. Taleb, F. Charra, and M.-P. Pileni, Phys. Rev. Lett. 84, 5840 (2000).

${ }^{2}$ J. J. Mock, M. Barbic, D. R. Smith, D. A. Schultz, and S. Schultz, J. Chem. Phys. 116, 6755 (2002).

${ }^{3}$ J. J. Xiao, J. P. Huang, and K. W. Yu, Phys. Rev. B 71, 045404 (2005).

${ }^{4}$ C. Voisin, N. Del Fatti, D. Christofilos, and F. Vallee, J. Phys. Chem. B 105, 2264 (2001).

${ }^{5}$ R. C. Jin, Y. W. Cao, C. A. Mirkin, K. L. Kelly, G. C. Schatz, and J. G. Zheng, Science 294, 1901 (2001).

${ }^{6}$ Y. Lu, G. L. Liu, and L. P. Lee, Nano Lett. 5, 5 (2005).

${ }^{7}$ J. Jiang, K. Bosnick, M. Maillard, and L. Brus, J. Phys. Chem. B 107, 9964 (2003).

${ }^{8}$ C. Hermann, Z. Kristallogr. 79, 186 (1931).

${ }^{9}$ J. Segall, J. Met. 9, 50 (1957).

${ }^{10}$ C. Mottet, J. Goniakowski, F. Baletto, R. Ferrando, and G. Treglia, Phase Transitions 77, 101 (2004).

${ }^{11}$ D. Reinhard, B. D. Hall, D. Ugarte, and R. Monot, Phys. Rev. B 55, 7868 (1997).

${ }^{12}$ M. R. Castell, Surf. Sci. 505, 1 (2002).

${ }^{13}$ T. Kubo and H. Nozoye, Surf. Sci. 542, 177 (2003).

${ }^{14}$ F. Silly and M. R. Castell, Phys. Rev. Lett. 94, 046103 (2005).

${ }^{15}$ F. Silly and M. R. Castell, J. Phys. Chem. B 109, 12316 (2005).

${ }^{16}$ F. Silly and M. R. Castell, Appl. Phys. Lett. 87, 053106 (2005).

${ }^{17}$ F. Silly and M. R. Castell, Appl. Phys. Lett. 87, 063106 (2005).

${ }^{18}$ J. A. Ascencio, M. Pérez, and M. José-Yacamán, Surf. Sci. 447, 73 (2000).

${ }^{19}$ S. Giorgio, J. Urban, and W. Kunath, Philos. Mag. A 60, 553 (1989).

${ }^{20}$ T. P. Martin, T. Bergmann, H. Gohlich, and T. Lange, Chem. Phys. Lett. 172, 209 (1990).

${ }^{21}$ T. P. Martin, T. Bergmann, H. Gohlich, and T. Lange, Chem. Phys. Lett. 176, 343 (1991).

${ }^{22}$ P. M. Ajayan and L. D. Marks, Nature (London) 338, 139 (1989).

${ }^{23}$ L. D. Marks, Philos. Mag. A 49, 81 (1984). 